\title{
BMJ Open User involvement in adolescents' mental healthcare: protocol for a systematic review
}

\author{
Petter Viksveen, ${ }^{1}$ Stig Erlend Bjønness, ${ }^{1}$ Siv Hilde Berg, ${ }^{2}$ \\ Nicole Elizabeth Cardenas, ${ }^{3}$ Julia Rose Game, ${ }^{3}$ Karina Aase, ${ }^{1}$ Marianne Storm ${ }^{1}$
}

To cite: Viksveen $P$, Bjønness SE, Berg SH, et al. User involvement in adolescents mental healthcare: protocol for a systematic review. BMJ Open 2017;7:e018800. doi:10.1136/ bmjopen-2017-018800

- Prepublication history and additional material for this paper are available online. To view these files, please visit the journal online (http://dx.doi org/10.1136/bmjopen-2017018800).

Received 27 July 2017 Revised 22 September 2017 Accepted 5 October 2017

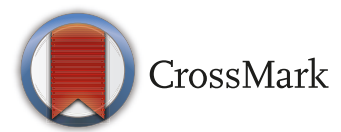

${ }^{1}$ SHARE-Centre for Resilience in Healthcare, Faculty of Health Sciences, University of Stavanger, Stavanger, Norway ${ }^{2}$ Department of Psychiatry, Stavanger University Hospital, Stavanger, Norway

${ }^{3}$ International Baccalaureate, St Olav videregående skole (High School), Stavanger, Norway

Correspondence to Dr Petter Viksveen; petter.viksveen@uis.no

\section{ABSTRACT}

Introduction User involvement has become a growing importance in healthcare. The United Nations state that adolescents have a right to be heard, and user involvement in healthcare is a legal right in many countries. Some research provides an insight into the field of user involvement in somatic and mental healthcare for adults, but little is known about user involvement in adolescents' mental healthcare, and no overview of the existing research evidence exists.

Methods and analysis The aim of this systematic review is to provide an overview of existing research reporting on experiences with and the effectiveness and safety issues associated with user involvement for adolescents' mental healthcare at the individual and organisational level. A systematic literature search and assessment of published research in the field of user involvement in adolescents' mental healthcare will be carried out. Established guidelines will be used for data extraction (Cochrane Collaboration guidelines, Strengthening the Reporting of Observational studies in Epidemiology and Critical Appraisal Skills Programme (CASP)), critical appraisal (Cochrane Collaboration guidelines and Pragmatic-Explanatory Continuum Indicator Summary) and reporting of results (Preferred Reporting Items for Systematic reviews and Meta-Analyses, Consolidated Standards of Reporting Trials and CASP). Confidence in the research evidence will be assessed using the Grading of Recommendations Assessment, Development and Evaluation approach. Adolescents are included as coresearchers for the planning and carrying out of this systematic review. This systematic review will provide an overview of the existing research literature and thereby fill a knowledge gap. It may provide various stakeholders, including decision-makers, professionals, individuals and their families, with an overview of existing knowledge in an underexplored field of research.

Ethics and dissemination Ethics approval is not required for this systematic review as we are not collecting primary data. The results will be published in a peer-reviewed journal and at conference presentations and will be shared with stakeholder groups.

\section{INTRODUCTION}

Mental health disorders affect a significant proportion of adolescents in countries all around the world. A meta-analysis of

\section{Strengths and limitations of this study}

- This is the first systematic review assessing user involvement for adolescents' mental healthcare.

- Established guidelines are used for data retrieval, data extraction, critical appraisal, data synthesis and reporting of results.

- Adolescents are involved as coresearchers through all phases of the systematic review.

- Wide inclusion criteria may represent challenges for synthesising the research evidence, although it will also provide a more extensive overview of the research literature.

- We expect to find limited research evidence in this field, which may also limit the extent to which we will be able to provide recommendations for clinical practice.

prevalence studies in 27 countries indicated that on average, $13.4 \%$ (95\% CI $11.3 \%$ to $15.9 \%$ ) in the age groups up to 18 years live with mental disorders. ${ }^{1}$ In many instances, these disorders result in functional impairment. The age group from 10 to 24 years represents $16 \%$ of all disability-adjusted life years of all age groups. ${ }^{2}$

According to the United Nations and the European Convention of Human Rights, children and adolescents have a right of access to high quality and safe healthcare services, and their views must be heard and considered in any matters affecting them. ${ }^{3} 4$ Although parents commonly have the authority to make decisions on behalf of their children, some national legislative frameworks (eg, in Canada, Ireland and the UK) also emphasise children's right to be heard and actively involved in decision-making concerning their own health. ${ }^{5}$ Adolescents at the age of 16 years are in some countries considered to be competent and have the right to consent to or refuse treatment. This may in some instances extend down to the age of 12 years (eg, in Ireland, Netherlands and the UK) or younger (eg, in Canada, New Zealand and 
South Africa), provided they are able to fully understand what the interventions involve. Some of the basic principles underpinning national legislation and regulations include respect, taking the adolescent seriously, encouraging cooperation and encouraging adolescents to take responsibility for their own health.

Patient-centred and person-centred or persondirected care with patient or service user involvement has become increasingly common in various fields of healthcare and at various levels. The term patient-centred care was initially described as focusing on the patient as a whole, including both physical and psychological aspects. ${ }^{6}$ It requires a more active and participating role on the part of the practitioner, compared with a more illness-oriented approach with uninvolved objective observation. In later years, the understanding of patient-centred care has taken into account service users' needs, priorities and expectations of healthcare services and can lead to reorganisation of services. ${ }^{7}$ A person-centred approach moves one step further by taking the whole person into consideration, including mental, emotional, spiritual and social needs, and in a person-directed approach, individuals take control of decisions affecting their own care. ${ }^{8}$

Person-centred or person-directed care provides a context for user involvement, which can be understood as engaging individual patients or users in processes of both planning and delivery of their own healthcare services (at the individual level), as well as their influence on provision of health services in general (at the organisational or institutional level), and for political decision-making processes. ${ }^{9}$ Users may here be understood as any person who is currently using or who may in the future use care services. This does de facto include any person/citizen. User involvement is encouraged through processes of collaboration with two-way communication, where professionals provide individuals with sufficient and adequate information, by eliciting their views, opinions, perceptions and perspectives. Such a form of user involvement requires reduction of power differentials between service users and healthcare professionals, where professionals must be willing to take users' views and wishes into account. ${ }^{10}$ When managed well, these processes may contribute to shared decision-making and selfdetermination for patients' healthcare services, provided within a context of person-centred or person-directed care. ${ }^{9}$

The active involvement of service users in healthcare decision-making has the potential to contribute to user empowerment and improvement of the quality and safety of healthcare systems. ${ }^{11}$ National legislation and regulation in many countries requires healthcare services to involve users in their own treatment, as well as at an institutional/organisational and political level. This includes various areas associated with mental healthcare, for example, in development of healthcare policies and strategies,${ }^{12}$ for implementation of clinical guidelines, ${ }^{13}$ in education of health professionals ${ }^{14}$ and for employment of healthcare staff and user-to-user panels. ${ }^{15}$ It has been suggested that users should be involved at every mental healthcare service level. ${ }^{16}$

Little is known about the existing research evidence reporting on user involvement for adolescents' mental healthcare. A literature search carried out in 2012 with an aim to assess the existing evidence of children's and adolescents' engagement in decision-making for their own healthcare identified only a handful of studies, in asthma, HIV, cancer, learning and behaviour problems and sun protection behaviours. ${ }^{17}$ No systematic review has focused specifically on user involvement in adolescents' mental healthcare.

Various definitions of adolescence exist. ${ }^{18-20}$ The term 'adolescence' comes from Latin and refers to 'growing up'. This phase of life involves the transition from childhood to adulthood. The time for the onset and conclusion of adolescence varies between individuals. In light of this, it may seem artificial to limit the age range when assessing mental healthcare services for adolescents. However, mental healthcare services for adolescents are commonly provided from the start of secondary school, typically around the age of 12 or 13 years. Moreover, in many countries, the age of 18 years is considered the legal transition into adulthood. The age range from 13 to 18 years corresponds to the Medical Subject Headings (MeSH) definition of adolescence (unique ID: D000293).

A number of important research questions need clarification, such as: what efforts are commonly put into place to encourage user involvement in adolescents' mental healthcare and how do they perceive such efforts? User involvement is commonly perceived to contribute positively to clinical treatment outcomes, and some research suggests that shared decision-making for individuals' own healthcare may be associated with improved health outcomes. ${ }^{21}{ }^{22}$ But what are the results of the overall research evidence for the effect of service user involvement on adolescents' mental health outcomes? Furthermore, how does user involvement affect patient safety? For example, some have found that adolescents who currently self-harm have reduced decision-making skills. ${ }^{23}$ Are particular measures needed to ensure the safety of this group when increasing user involvement?

\section{Objectives}

This systematic review aims to provide an overview of existing research reporting on experiences with and the effectiveness and safety issues associated with user involvement for adolescents' mental healthcare.

The objectives of this review are:

A. To assess the experiences with user involvement for adolescents' mental healthcare at the individual and organisational level. 
B. To assess the effectiveness of user involvement for adolescents' mental healthcare at the individual and organisational level.

C. To assess safety issues associated with user involvement for adolescents' mental healthcare at the individual and organisational level.

\section{METHODS}

We will use predefined eligibility criteria and search strategies and guidelines for data extraction, critical appraisal, data synthesis and reporting of results.

\section{Eligibility criteria}

The inclusion criteria for this systematic review are: studies reporting on experiences with, effectiveness of and safety issues associated with user involvement (intervention) in the planning, delivery and development of mental healthcare for adolescents (participants). Mental healthcare may include preventive or therapeutic interventions for diagnosed conditions or self-reported problems. User involvement may be aimed at the individual level (for adolescents' own mental healthcare) or at the organisational or institutional level (to improve mental healthcare services/institutions). It is unlikely that the effectiveness of user involvement has been tested in randomised controlled trials. We will therefore include non-randomised trials and studies (NRS). Additionally, we will not limit the literature search to any specific research methods, as this may lead to missing studies in systematic reviews including NRS. ${ }^{24}$ Studies may include any research design (qualitative or quantitative) used to answer the research questions of this review and may or may not include comparators/control groups (control). Outcomes will be reported according to the original trials and studies. Adolescents' user involvement may have been reported by adolescents, their caretakers, health professionals or other stakeholder groups. Adolescents will be defined as the age group from 13 to 18 years (MeSH unique ID: D000293). Studies reporting on adolescents and children or adults will be included if data for adolescents can be extracted and analysed separately. Results or the peer-reviewed and the grey literature will be reported separately. Exclusion criteria: debate, commentaries, editorials and studies reporting on children (age below 13 years) or adults (age above 18 years). Languages will be limited to English, French, German, Danish, Norwegian and Swedish. The literature will be limited to the last 15 years $(2002-2017)$.

\section{Search strategy}

A systematic search of the following databases will be carried out: Academic Search Premier, British Nursing Index, Cumulative Index to Nursing and Allied Health Literature, Cochrane Library, Excerpta Medica Database, Medical Literature Analysis and Retrieval System Online (MEDLINE), PubMed, PsycINFO, Scopus, SocINDEX, SveMed+ and Web of Science (complete list in box). Reference lists of included studies will be hand

\section{Box Literature sources}

Databases (with dates of coverage)

- Academic Search Premier (2002-2017)

- BNI: British Nursing Index (2002-2017)

- CINAHL: Cumulative Index to Nursing and Allied Health Literature (2002-2017)

- Cochrane Library (2002-2017)

- EMBASE: Excerpta Medica Database (2002-2017)

- Medical Literature Analysis and Retrieval System Online (MEDLINE): National Library of Medicine (2002-2017)

- PubMed*: National Library of Medicine (2016-2017)

- PsycINF0: American Psychological Association (2002-2017)

- Scopus (2002-2017)

- SocINDEX: Database for Sociological Research (2002-2017)

- SveMed+: Medical data by the Karolinska Institute Library (2002-2017)

- Web of Science (2002-2017)

\section{Other sources}

- Google Scholar

- User groups

- Experts and researchers

*PubMed search will be limited to the last 2 years, as older titles are likely to be covered by MEDLINE.

searched for identification of additional titles. The grey literature will be searched using an advanced Google Scholar search limited to the first 50 results for each search string and through user/interest groups, experts and researchers in the field. An expert university librarian has been consulted as part of planning the literature search strategy. A draft search strategy for one electronic database (MEDLINE) is presented in online supplementary appendix $\mathrm{A}$.

A wide range of search terms will be used in order to identify relevant literature, as user involvement may include a whole range of different activities. Search strategies aimed at maximising sensitivity and specificity will be customised to databases, where possible using MeSH/ subject terms, explode function, wild card symbols and Boolean operators. A combination of groups of search terms will be used, including the service user group (adolescents), the field of health (psychiatry/mental health) and the field of research (involvement), as presented in table 1 .

A minimum of two reviewers will screen titles and abstracts to determine inclusion/exclusion of articles. All potentially relevant full text articles will be read and assessed by at least two reviewers according to the inclusion/exclusion criteria. In the event of continued doubt, a third reviewer will be included for consensus or majority vote decisions. Reasons for exclusion will be logged. Endnote (V.X8) will be used to manage data records.

\section{Data extraction}

Data will be extracted and input by one reviewer into an Excel spreadsheet using the Cochrane Consumers and Communication Review Group's data extraction template 
Table 1 Search terms

\section{Subject/MeSH terms}

\begin{tabular}{|c|c|c|c|c|}
\hline \multirow{2}{*}{\multicolumn{2}{|c|}{$\begin{array}{l}\text { User group and field of health } \\
\text { Adolescent psychiatry }\end{array}$}} & \multicolumn{3}{|l|}{ Field of research } \\
\hline & & Clinical decision-making & Comr & \\
\hline \multicolumn{2}{|c|}{ Adolescent psychology } & \multicolumn{2}{|l|}{ Consumer participation } & king \\
\hline & \multicolumn{2}{|l|}{ Cooperative behaviour } & Decision-making, organisational \\
\hline & & \multicolumn{2}{|l|}{ Information sharing } & Information dissemination \\
\hline & & Patient participation & & Personal autonomy \\
\hline & & Public opinion & & Self-determination \\
\hline \multicolumn{5}{|c|}{ Terms used in search of title } \\
\hline User group & Field of health & \multicolumn{3}{|l|}{ Field of research } \\
\hline Adolescents & Mental & Autonomy & Client-centred & Collaboration \\
\hline Teenagers & Psychology & Consultation & Contribution & Decision-making \\
\hline \multirow[t]{5}{*}{ Youth } & Psychiatry & Empowerment & Engagement & Governance \\
\hline & & Inclusion & Information sharing & Involvement \\
\hline & & Mutual agreement & Negotiation & Opinions \\
\hline & & Patient-centred & Participation & Partnership \\
\hline & & Perspectives & Peer support & Self-determination \\
\hline
\end{tabular}

MeSH, Medical Subject Headings.

for trials, ${ }^{25}$ the Strengthening the Reporting of Observational Studies in Epidemiology (STROBE) statement checklist for cohort, case-control and cross-sectional studies $^{26}$ and the Critical Appraisal Skills Programme (CASP) for qualitative studies. ${ }^{27} \mathrm{~A}$ second reviewer will check the accuracy of input data. Crossreferences to article publications may be used, but authors of original trials and studies will not be contacted for clarification as this may lead to too optimistic and biased responses. ${ }^{28}$ For studies reporting on the effectiveness of interventions, a main outcome will be identified as defined by the authors of the original article. Reviewers will decide on which outcome measure to report in the event that no main outcome has been defined by study authors, as well as any additional outcomes of importance to users.

\section{Critical appraisal}

Including studies and trials using various research methods could potentially result in misleading effect estimates. ${ }^{24}$ To avoid this, we will use the Cochrane Collaboration's guidelines to assess risk of bias at the outcome level. ${ }^{28}$ We will assess the risk of selection bias, performance bias, detection bias, attrition bias and reporting bias. Particular attention will be paid to the potential influence of confounding factors for NRS, as suggested by the Cochrane Collaboration. ${ }^{24}$ Moreover, we will assess the risk of metabias by searching for unpublished studies in the grey literature, by comparing protocols articles with results articles and by assessing methods and results sections of individual study articles. If there is a sufficient number of studies (minimum of 10) with variation in trial sizes (at least one medium or large), funnel plot symmetry together with a regression analysis will be assessed to consider risk of publication bias, as suggested by Sterne $e t a l^{29}$ However, whether a trial is free of bias does not address the question of its applicability and generalisability to end users, including patients, clinicians and policy-makers. ${ }^{30}$ We will therefore assess the external validity of trials using the Pragmatic-Explanatory Continuum Indicator Summary tool. ${ }^{31}$ CASP will be used for assessing qualitative studies. ${ }^{27}$

The confidence in the evidence resulting from the identified research literature will be assessed using the Grading of Recommendations Assessment, Development and Evaluation approach. ${ }^{32}$ This will include assessment of the risk of bias, inconsistency, indirectness, imprecision, publication bias and factors increasing the confidence in the effect of each outcome.

\section{Data synthesis}

The extent to which data will be synthesised will depend on the degree of homogeneity of included studies. We will determine consistency of the evidence by assessing the variability of trials. Particular attention will be paid to clinical aspects (eg, interventions and clinical conditions) and research methods. The effect of heterogeneity will be quantified using the $\mathrm{I}^{2}$ statistic to calculate variability across trials. ${ }^{33}$

In the event of sufficient homogeneity, results of randomised trials will be presented collectively through a meta-analysis, presenting effect estimates with SEs or CIs. A summary statistic will be calculated for the main outcome in each trial, determining the risk ratio for dichotomous outcomes and standardised mean differences for continuous outcomes (both with 95\% CIs). A weighted average will be calculated to determine the 
overall treatment effect in the included trials. We expect at least some heterogeneity between trials and will therefore apply a random-effects model to estimate the mean of the distribution of effects. Analysis of subgroups will be considered for specific clinical conditions (eg, depression, anxiety or psychosis), for particular research methods (eg, randomised controlled trials, cluster randomised trials or non-randomised studies and trials) and if the $\mathrm{I}^{2}$ statistic supersedes $40 \%$. For groups of NRS applying similar study designs, summarised adjusted effect estimates will be presented (controlling for confounding factors) as suggested by Reeves $e t a l .^{24}$ Only a narrative summary will be developed in the event of considerable heterogeneity $\left(\mathrm{I}^{2}>75 \%\right)$. Dependent on a sufficient number of included studies, qualitative studies will be synthesised. ${ }^{34}$ Some flexibility is needed with regard to choice of the most suitable approach for the qualitative synthesis as this will depend on the available research evidence, in particular with regard to the quality and heterogeneity of studies. We will therefore make a final decision to determine the synthesis approach after collection of data but will aim at applying either a textual narrative synthesis or a thematic analytical synthesis approach..$^{35} 36$

\section{Reporting results}

The Preferred Reporting Items for Systematic reviews and Meta-Analyses statement and flow diagram will be used to report the result of literature searches. ${ }^{37}$ Results of randomised controlled trials will be reported according to the Consolidated Standards of Reporting Trials statement, ${ }^{38}$ for observational studies the STROBE statement ${ }^{26}$ and for qualitative studies the CASP checklist. ${ }^{27}$ Results of all studies will be presented in tables, providing information on study design, results and quality of evidence. Separate tables will be provided in the event of different subgroups of patients. We will report results collectively for comparative (sufficiently homogeneous) studies, grouped according to their associated risk of bias. Results will be presented separately for randomised controlled trials and NRS. Moreover, the nature of the data will also warrant presentation of results according to research methods (eg, results of qualitative and quantitative studies will be reported separately). Information on methodological decisions made or modified after data collection will be reported. Moreover, we will report involvement of user/interest groups, experts and researchers.

\section{Coresearchers and user involvement}

Two adolescent coresearchers (NEC and JRG) have been involved in developing and writing the protocol for the systematic review and will be involved in the systematic review process. They were invited to participate in the systematic review as part of the InvolveMENT research project, which they are already involved in. The InvolveMENT project aims to assess factors affecting adolescents' mental health and to develop and assess the effectiveness of an intervention. Mental health organisations have also been invited to the planning (but have not yet participated), carrying out and dissemination of the results of this systematic review.

The adolescent coresearchers' role and contribution

We have participated in the process of developing the systematic review, and we will contribute in the review process and for publishing the results. We have agreed to be asked for our opinion on articles the other researchers consider, including their evaluation of the content and quality of the articles and how results will be reported. We will also publish a lay summary of the results on a Facebook site we are setting up for the research project.

We expressed our interest in being part of the InvolveMENT project team following a presentation that was given at our high school in January 2017 by a researcher (PV). Since then we have had monthly meetings and have been introduced to the field of adolescent mental health research, research terminology and methodology and the systematic review process. We have also participated at a university workshop to learn the basics of systematic reviews and literature searches. We have asked not to be referred to as 'user representatives', as we are not participants in the research, and the term can carry stigma. Instead, we asked to be referred to as 'adolescent representatives'. It was later decided that we should be redefined as 'coresearchers' as we became more actively involved in the research. For example, we have carried out a questionnaire survey in cooperation with one of the researchers $(\mathrm{PV})$ to learn about the prevalence of mental disorders and stress in teenagers and their use of mental healthcare services. This research was started in June this year and is still ongoing. We plan to present the results to the students who took part in the survey, teachers and school nurses, and we hope that it will help to reduce some of the stigma surrounding mental health and to improve mental health services.

\section{DISCUSSION}

User involvement has become a priority in healthcare systems in many countries, as it is considered a citizen right to be involved in decisions affecting one's own healthcare. Although several systematic reviews have summarised existing evidence in user involvement in health research for adults in general or in areas such as safety and education of mental health professionals, ${ }^{39-41}$ no review has been published assessing user involvement for adolescents' mental healthcare. This systematic review therefore aims to fill an existing knowledge gap. It will provide an insight into users' experiences, effectiveness of and safety issues associated with user involvement at an individual level for adolescents' own mental healthcare and at an organisational or institutional level for improvement of mental healthcare services and institutions. It may thereby provide information that can be valuable for several stakeholder groups, such as patients and their families, healthcare providers, clinicians and decision-makers, as well as for developing research 
strategies to further knowledge in an underexplored field of research. This may include knowledge about ways in which users are involved in decision-making affecting their own mental healthcare and the influence of such involvement on their mental health outcomes. It can also provide information on user involvement affecting delivery and mental healthcare service policy decisions.

There are some potential limitations to this systematic review. There is considerable variation in the literature with regard to used terminology relating to user involvement. This contributes to a risk of missing relevant studies. We have, in an attempt to capture the relevant research literature, included several search strategies using a variety of search terms. Moreover, not including search terms relating to specific mental diagnoses contributes to a risk of missing relevant studies. We are however interested in reviewing the mental health research literature overall and not focus on a limited number of specific conditions. Inclusion of the breath of different diagnoses would also contribute to an unmanageable number of titles to screen. Moreover, we hope to capture most of the literature relevant to adolescents, although research carried out with varying age groups (single studies reporting on both children and adolescents or adolescents and young people) may limit the breath of literature we will include in our review. The definition of 'adolescents' varies within the context of different national legislations and individual researchers' and clinicians' understanding. We have limited this literature review to adolescents in the age range from 13 to 18 years. It could be argued for a different age span for adolescents, for example, using Unicef's definition and the results of this review will not be applicable to children under the age of 13 years or young persons above the age of 18 years. We do however think that our search strategy will help us to capture most of the relevant literature, that is, of relevance to user involvement in adolescents' mental healthcare.

Another limitation of this systematic review is the restriction to only six languages. Although we expect to capture most of the published research literature through English language articles, and although some evidence suggests reduced importance of non-English language articles for assessing the effectiveness of interventions, ${ }^{42}$ we cannot exclude the possibility that significant literature has been published in other languages. We have selected six languages in an attempt to limit the gaps in the research evidence collected through our review. Our choice of languages is partly due to the strong tradition user involvement has in some of these countries but is nevertheless limited by our available resources. The inclusion of a wide range of databases increases the likelihood of capturing most of the relevant literature in this field of research. Moreover, the inclusion of a database more specifically covering the Nordic literature will increase the chance of also capturing the evidence published in these countries.
Our systematic review team includes adolescent coresearchers. Others have suggested that public or user involvement may contribute significantly to various stages of the systematic review process. ${ }^{43-47}$ It has however been pointed out that such involvement can in some cases be tokenistic, ${ }^{47}$ and that power differentials may affect the usefulness of such involvement. ${ }^{43}$ We argue that the involvement of adolescents as coresearchers may contribute by strengthening the usefulness of the review for different stakeholder groups, in particular for adolescents themselves.

Acknowledgements Many thanks to Grete Mortensen, librarian at the University of Stavanger, for useful advice on strategies for our literature search.

Contributors PV is the guarantor. All authors contributed to the development of the manuscript draft. PV, SEB, SHB, KA and MS contributed to the development of the search and assessment strategies. All authors read, provided feedback and approved the final manuscript.

Competing interests None declared.

Provenance and peer review Not commissioned; externally peer reviewed.

Open Access This is an Open Access article distributed in accordance with the Creative Commons Attribution Non Commercial (CC BY-NC 4.0) license, which permits others to distribute, remix, adapt, build upon this work non-commercially, and license their derivative works on different terms, provided the original work is properly cited and the use is non-commercial. See: http://creativecommons.org/ licenses/by-nc/4.0/

(c) Article author(s) (or their employer(s) unless otherwise stated in the text of the article) 2017. All rights reserved. No commercial use is permitted unless otherwise expressly granted.

\section{REFERENCES}

1. Polanczyk GV, Salum GA, Sugaya LS, et al. Annual research review: a meta-analysis of the worldwide prevalence of mental disorders in children and adolescents. J Child Psychol Psychiatry 2015;56:345-65.

2. Gore FM, Bloem PJ, Patton GC, et al. Global burden of disease in young people aged 10-24 years: a systematic analysis. Lancet 2011;377:2093-102.

3. European Union (EU) and Council of Europe (CoE). Handbook on European law relating to the rights of the child. European Union Agency for Fundamental Rights and Council of Europe, 2015:ISBN 978-92-871-9917-1.

4. United Nations. The UN Convention on the Rights of the Child, United Nations. 1989, (reprinted June 2010). articles art. 12, 24. ISBN 0-9553005-4-1.

5. Lansdown G. Every child's right to be heard. A resource guide on the UN Committee on the Rights of The Child general comment no.12. Save the Children UK on behalf of Save the Children and UNICEF, 2011. https://www.unicef.org/french/adolescence/files/Every_Childs_ Right_to_be_Heard.pdf (accessed 16 Nov 2016).

6. Balint M, Ball DH, Hare ML. Training medical students in patientcentered medicine. Compr Psychiatry 1969;10:249-58.

7. Lutz BJ, Bowers BJ. Patient-centered care: understanding its interpretation and implementation in health care. Sch Inq Nurs Pract 2000;14:165-82.

8. Lines LM, Lepore M, Wiener JM. Patient-centered, person-centered, and person-directed care: They are not the same. Med Care 2015;53:561-3.

9. Storm M, Edwards A. Models of user involvement in the mental health context: intentions and implementation challenges. Psychiatr Q 2013;84:313-27.

10. Borg M, Karlsson B, Kim HS. User involvement in community mental health services-principles and practices. J Psychiatr Ment Health Nurs 2009;16:285-92.

11. Dent M, Pahor M. Patient involvement in Europe-a comparative framework. J Health Organ Manag 2015;29:546-55.

12. Semrau M, Lempp H, Keynejad R, et al. Service user and caregiver involvement in mental health system strengthening in low- and middle-income countries: systematic review. BMC Health Serv Res 2016;16:79. 
13. Moreno EM, Moriana JA. User involvement in the implementation of clinical guidelines for common mental health disorders: a review and compilation of strategies and resources. Health Res Policy Syst 2016;14:61.

14. Scammell J, Heaslip V, Crowley E. Service user involvement in preregistration general nurse education: a systematic review. J Clin Nurs 2016;25:53-69.

15. Baxter S, Clowes M, Muir D, et al. Supporting public involvement in interview and other panels: a systematic review. Health Expect 2017;20:807-17.

16. Browne G, Lakeman R, O'Brien AP, et al. Service users on interview panels in mental health. Int J Ment Health Nurs 2015;24:281-2.

17. Feenstra B, Boland L, Lawson ML, et al. Interventions to support children's engagement in health-related decisions: a systematic review. BMC Pediatr 2014;14:109.

18. Lamb C, Murphy M. The divide between child and adult mental health services: points for debate. Br J Psychiatry Suppl 2013;54:s41-4

19. McGorry P, Bates T, Birchwood M. Designing youth mental health services for the 21st century: examples from Australia, Ireland and the UK. Br J Psychiatry Suppl 2013;54:s30-5.

20. United Nations Children's Fund (UNICEF). The State of the World's Children. Adolescence: An Age of Opportunity. UNICEF 2011.

21. Langer DA, Jensen-Doss A. Shared decision-making in youth mental health care: using the evidence to plan treatments collaboratively. J Clin Child Adolesc Psychol 2016;2:1-11.

22. Shay LA, Lafata JE. Where is the evidence? A systematic review of shared decision making and patient outcomes. Med Decis Making 2015;35:114-31.

23. Oldershaw A, Grima E, Jollant F, et al. Decision making and problem solving in adolescents who deliberately self-harm. Psychol Med 2009;39:95-104.

24. Reeves BC, Deeks JJ, Higgins JPT, et al. Chapter 13: Including non-randomized studies. In: Higgins JPT, Green S, eds. Cochrane Handbook for Systematic Reviews of Interventions Version 5.1.0 (updated March 2011). The Cochrane Collaboration, 2011. http:// www.handbook.cochrane.org (accessed 5 May 2017).

25. Cochrane Consumers and Communication Review Group. Data extraction template for included studies. Version 1.7, updated 9 June, 2016. http://cccrg.cochrane.org/author-resources (accessed 24 Nov 2016).

26. Vandenbroucke JP, von Elm E, Altman DG, et al. Strengthening the Reporting of Observational Studies in Epidemiology (STROBE): explanation and elaboration. Int J Surg 2014;12:1500-24.

27. CASP UK, Critical Appraisal Skills Programme (CASP). Qualitative research checklist 31.05.13. http://www.casp-uk.net/casp-toolschecklists (accessed 4 May 2017).

28. Higgins JPT, Altman DG, Sterne JAC. Assessing risk of bias in included studies. In: Higgins JPT, Green S, eds. Cochrane Handbook for Systematic Reviews of Interventions, Version 5.1.0. The Cochrane Collaboration, 2011. updated march 2011.

29. Sterne JA, Gavaghan D, Egger M. Publication and related bias in meta-analysis: power of statistical tests and prevalence in the literature. J Clin Epidemiol 2000;53:1119-29.
30. Treweek S, Zwarenstein M. Making trials matter: pragmatic and explanatory trials and the problem of applicability. Trials 2009;10:37.

31. Thorpe KE, Zwarenstein M, Oxman AD, et al. A pragmaticexplanatory continuum indicator summary (PRECIS): a tool to help trial designers. CMAJ 2009;180:E47-E57.

32. Guyatt GH, Oxman AD, Schünemann HJ, et al. GRADE guidelines: a new series of articles in the Journal of Clinical Epidemiology. J Clin Epidemiol 2011;64:380-2.

33. Higgins JP, Thompson SG, Deeks JJ, et al. Measuring inconsistency in meta-analyses. BMJ 2003;327:557-60.

34. Barnett-Page E, Thomas J. Methods for the synthesis of qualitative research: a critical review. BMC Med Res Methodol 2009;9:59.

35. Lucas PJ, Baird J, Arai L, et al. Worked examples of alternative methods for the synthesis of qualitative and quantitative research in systematic reviews. BMC Med Res Methodol 2007;7:4.

36. Thomas J, Harden A. Methods for the thematic synthesis of qualitative research in systematic reviews. BMC Med Res Methodol 2008;8:45.

37. Moher D, Liberati A, Tetzlaff J, et al. PRISMA Group. Preferred reporting items for systematic reviews and meta-analyses: the PRISMA statement. PLoS Med 2009;6:e1000097.

38. Schulz KF, Altman DG, Moher D. CONSORT Group. CONSORT 2010 statement: updated guidelines for reporting parallel group randomised trials. BMJ 2010;340:c332

39. Brett J, Staniszewska S, Mockford C, et al. Mapping the impact of patient and public involvement on health and social care research: a systematic review. Health Expect 2014;17:637-50.

40. Hall J, Peat M, Birks Y, et al. Effectiveness of interventions designed to promote patient involvement to enhance safety: a systematic review. Qual Saf Health Care 2010;19:e10.

41. Happell B, Byrne L, McAllister M, et al. Consumer involvement in the tertiary-level education of mental health professionals: a systematic review. Int J Ment Health Nurs 2014;23:3-16.

42. Galandi D, Schwarzer $G$, Antes $G$. The demise of the randomised controlled trial: bibliometric study of the German-language health care literature, 1948 to 2004. BMC Med Res Methodol 2006;6:30.

43. Boote J, Baird W, Sutton A. Public involvement in the systematic review process in health and social care: a narrative review of case examples. Health Policy 2011;102:105-16.

44. Crawford MJ, Rutter D, Manley C, et al. Systematic review of involving patients in the planning and development of health care. BMJ 2002;325:1263

45. Pollock A, Campbell P, Baer G, et al. User involvement in a Cochrane systematic review: using structured methods to enhance the clinical relevance, usefulness and usability of a systematic review update. Syst Rev 2015;4:55.

46. Smith E, Donovan S, Beresford P, et al. Getting ready for user involvement in a systematic review. Health Expect 2009;12:197-208.

47. Coon JT, Gwernan-Jones R, Moore D, et al. End-user involvement in a systematic review of quantitative and qualitative research of non-pharmacological interventions for attention deficit hyperactivity disorder delivered in school settings: reflections on the impacts and challenges. Health Expect 2016;19:1084-97. 